\title{
Uso de Muhlenbergia macroura (Poaceae:Eragrostideae) en la región de Perote, Veracruz, México
}

La conocida importancia económica que tienen las gramíneas para el hombre, desde el punto de vista agrícola, ganadero e industrial, se ha señalado en innumerables ocasiones. Sin embargo, se ha estudiado poco el aspecto del uso y manejo tradicional de estas plantas por las comunidades indígenas.

Tal es el caso de Muhlenbergia macroura (H.B.K.) Hitchc. o raíz de zacatón, especie nativa de Mesoamérica y que se ha utilizado en la fabricación de escobetas, escobas y cepillos desde hace más de 100 años (Avila, 1980). Esta especie, así como otras de los géneros Stipa y Festuca, forman parte natural de las praderas alpinas y subalpinas, comúnmente llamados zacatonales del Eje Neovolcánico Transversal (Miranda y Hernández, 1963) o tambien se puede localizar en bosque de pino, bosque de encino, bosque de pinoencino y en bosque de oyamel, a una altitud de 1800 a $4000 \mathrm{~m}$. Su distribución se ha registrado en los estados de: Aguascalientes, Chiapas, Chihuahua, Distrito Federal, Durango, Estado de México, Hidalgo, Jalisco, Michoacán, Morelos, Oaxaca, Puebla, Querétaro, San Luis Potosí, Tlaxcala y Veracruz (Beetle, 1977). Recibe diversos nombres comunes de acuerdo a las regiones donde crece, tales como: zacatón (Estado de México), surumuta en lengua tarasca (Michoacán), zacate malinali o malinali en lengua náhuatl (Morelos), cobachita o gobachita en lengua zapoteca (Oaxaca), zacate de escoba (San Luis Potosí) y raíz de zacatón (Veracruz) (Martínez, 1979; Mejía y Dávila, 1992).

El uso más común de la raíz de zacatón ha sido la elaboración de escobetas, escobas y cepillos (Jackson, 1887; Martínez, 1979), otro uso ha sido como forraje (Díaz,P. 1976); ha sido recomendado para la fabricación de papel (Nieto, 1985), como medicinal (Díaz, G. 1976), en la fabricación de escobas de popote, donde se utilizan los tallos florales o también se pueden utilizar los desperdicios de la raíz como abono (Martínez, 1959).

Muhlenbergia macroura es una hierba perenne, que forma grandes macollos de $60-130 \mathrm{~cm}$ de altura, su raíz es fibrosa, de $20-30 \mathrm{~cm}$ de alto está constituida de numerosas fibras gruesas de diferente longitud, rizadas y ásperas, de aproximadamente $1 \mathrm{~mm}$ de diámetro, con una capa cortical (llamada comunmente camisilla); las hojas son lineares, estrechas y planas; la inflorescencia es una panícula densa, erecta, de $15-40 \mathrm{~cm}$ de longitud, de color verdegrisáceo, las espiguillas son aproximadamente de $4 \mathrm{~mm}$ de longitud. 
En el estado de Veracruz se ha colectado Muhlenbergia macroura en el extremo centro oeste del estado de Veracruz (que forma parte del Eje Neovolcánico Transversal), principalmente en la región de Perote, localizada entre las coordenadas $19^{\circ} 27^{\prime}$ y $19^{\circ} 41^{\prime}$ latitud norte y $97^{\circ} 07^{\prime}$ y $97^{\circ} 17^{\prime}$ longitud oeste, abarcando parcialmente los municipios de Perote y Villa Aldama. Gerez (1982) menciona que en el valle y faldas del Cofre de Perote y cerca de los límites con Puebla, se ecuentra una extension de $51.2 \mathrm{~km}$ de zacatonales, así mismo menciona que los asentamientos humanos de esta zona utilizaban algunas plantas en sus actividades artesanales; tal es el caso de Muhlenbergia macroura (sin especificar su uso) la cual llega a ser en 1946 una de las actividades principales la extracción de raíz de zacatón.

Posteriormente Sandoval (1984) hace referencia que dentro de las plantas silvestres útiles en la Sierra de Mastaloyan (región semiárida del estado de Veracruz y húmeda del estado de Puebla), se utiliza la raíz de zacatón para la elaboración de escobetas.

Con estos antecedentes nació la inquietud de conocer el uso tradicional de Muhlenbergia macroura en la región de Perote, Veracruz, así como la importancia económica que representa esta actividad en donde es utilizada.

Como primer paso se consultó los bancos de datos de Flora de Veracruz y el de Plantas Utiles del Instituto de Ecología, A.C. con lo cual se localizaron 11 lugares en la región de Perote donde ha sido colectada esta especie y solo en dos de estas localidades se encontró que esta especie es utilizada. También se realizaron visitas a los principales mercados de Xalapa, Perote y Las Vigas para tratar de localizar otros lugares en donde se utilizara la raíz de zacatón. Como resultado se obtuvieron dos localidades donde utilizaban Muhlenbergia macroura las cuales fueron: Cruz Blanca y Tenextepec. (fig. 1).

Se prepararon ejemplares de herbario así, como visitas periodicas a las localidades para hacer las entrevistas y recabar la información del proceso de extracción de la materia prima y elaboración de los productos artesanales como escobetas, escobas y cepillos.

Como resultado se obtuvo que la extracción de la raíz de zacatón la realizan del medio natural y forma parte complementaria de las actividades agrícolas y ganaderas de las personas de la localidad de Cruz Blanca, por lo cual esta actividad esta combinada con la época de cultivo del maíz y solo elaboran escobetas en los meses de marzo, abril y parte de mayo. En el caso de Tenextepec la elaboración de estos productos se llevo a cabo todo el año, ya que la comunidad depende económicamente de la venta de estos productos artesanales.

El proceso de extracción y elaboración es similar en las dos localidades. Desde la extracción de la raíz hasta la venta de la escobeta o escoba, se necesita invertir una semana de trabajo. Esta labor la realiza el hombre, aunque la mujer es generalmente la encargada de realizar la venta y distribución de la mercancia en los diferentes mercados. 
NOTAS BOTANICAS

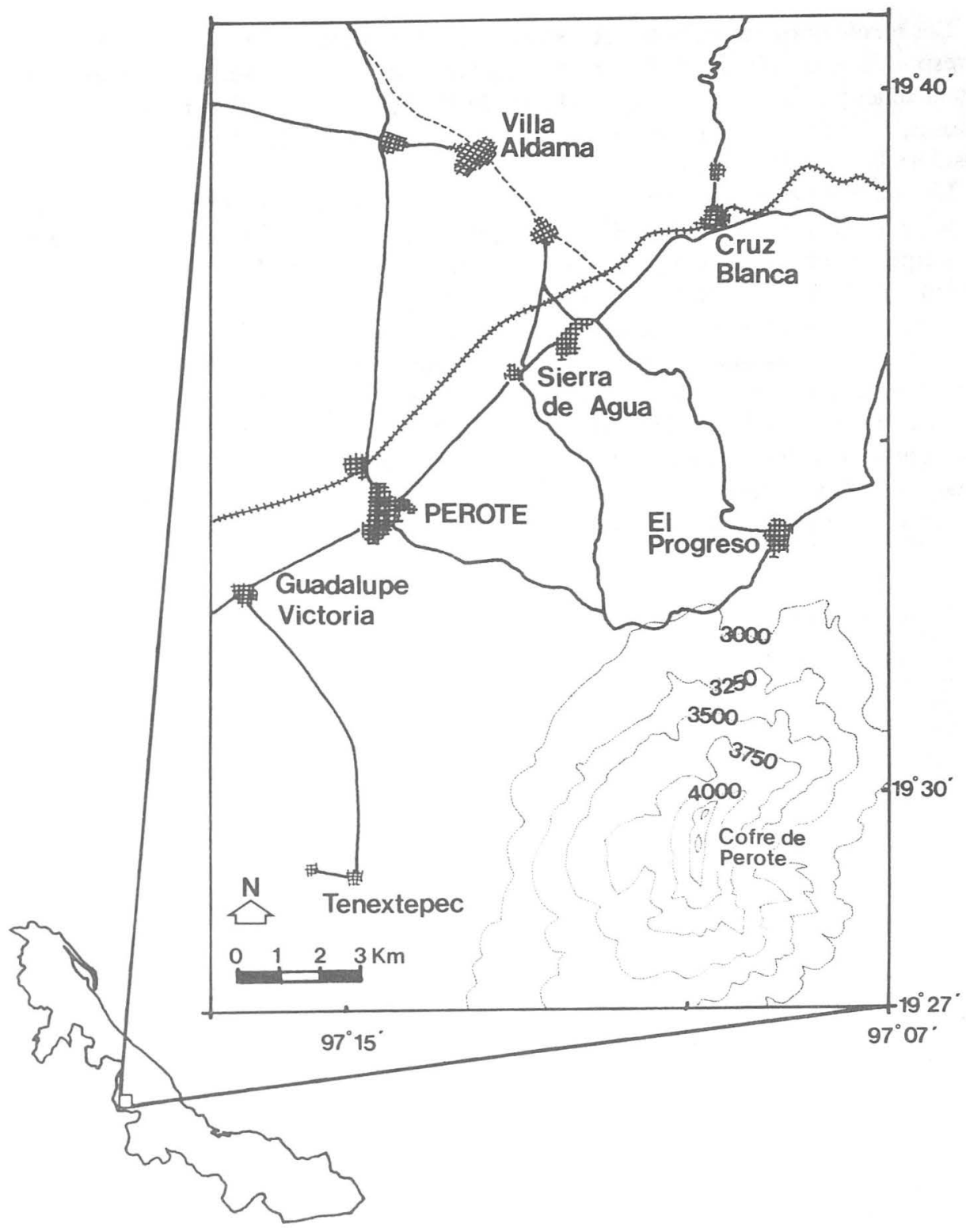

Fig. 1. Localización geográfica de las comunidades estudiadas 
Los recolectores del zacatón reconocen dos clases de raíz, a) la fina o derecha, que corresponde a la raíz de $30-40 \mathrm{~cm}$ de longitud, más o menos recta y con escasas ondulaciones y b) la raíz"china", que es la raíz de $15-20 \mathrm{~cm}$ de longitud, con ondulaciones sinuosas, las que ocasionan que la raíz se rompa fácilmente y, por consiguiente, es considerada de mala calidad.

La raíz la obtienen del valle o faldas del Cofre de Perote, de preferencia en lugares con suelo fino y arenoso. Se elige el macollo más grande de zacatón y dejan los macollos más pequeños para que se sigan desarrollando. Con el gorguz (fig. 2 A) se escarba alrededor de la planta y cuando el gorguz está a una profundidad aproximadamente de $40-50 \mathrm{~cm}$, se levanta la planta con la tlalacha (fig. 2 B), cuidando de no estropear la raíz. Con esta misma herramienta se hace palanca para remover la raíz. Este paso es importante ya que se necesita de mucho cuidado y práctica para no romper las raices.

Una vez extraída la planta se sacude contra una piedra, con el fin de quitarle la mayor parte de la tierra y basurillas. Reunida cierta cantidad de raíz se corta el follaje, el cual, cuando está tierno, es aprovechado como alimento para el ganado caprino. Todo este proceso lo realiza el jefe de familia y en ocasiones con la ayuda del hijo mayor.
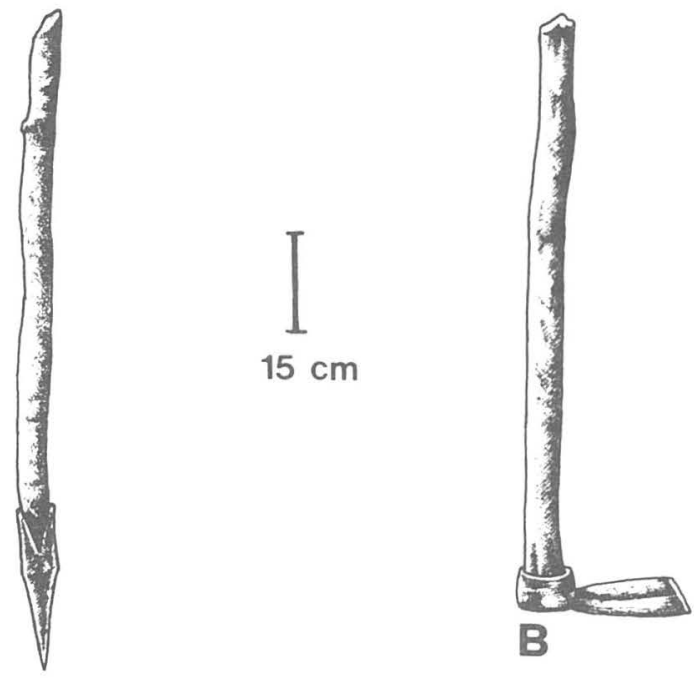

A

Fig. 2 A gorguz, B tlalacha 
Después de la extracción, se lleva a cabo la transformación de la raíz. Este es uno de los pasos más importantes dentro de la comercialización, ya que el tratamiento que se le proporciona a la raíz hace que su calidad (en cuanto aspecto) aumente.

Después de sacudir la raíz, se remoja en agua durante 24 horas, posteriormente, se azota o apalea contra una piedra o se le pega con un palo para que se le caiga la tierra y se desprenda la camisilla. A la vez también se van quitando la raíz china y la basurilla que vaya quedando. Este proceso se lleva a cabo durante dos días consecutivos. Al pasar por este proceso la raíz se limpia y cambia de un color oscuro a uno amarillento.

La raíz se pone a secar en el solar de la casa extendiendo el matojo de raíz para que penetre el sol y el secado sea uniforme.

Una vez seca la raíz, se procede a la elaboración o amarre de escobetas y escobas. En este proceso se utiliza alambre y una hoz para emparejar las puntas de las escobetas. Para las escobas se utiliza un palo de pino colorado, Pinus patula Schlecht. \& Cham., el cual sirve de mango.

De la raíz de un macollo se llegan a obtener 3 o 4 escobetas, o una escoba y una escobeta. Los productos obtenidos del zacatón (fig. 3) se distribuyen en los mercados de Perote, Las Vigas y Xalapa, y en algunas ocasiones en el mercado de Veracruz. El

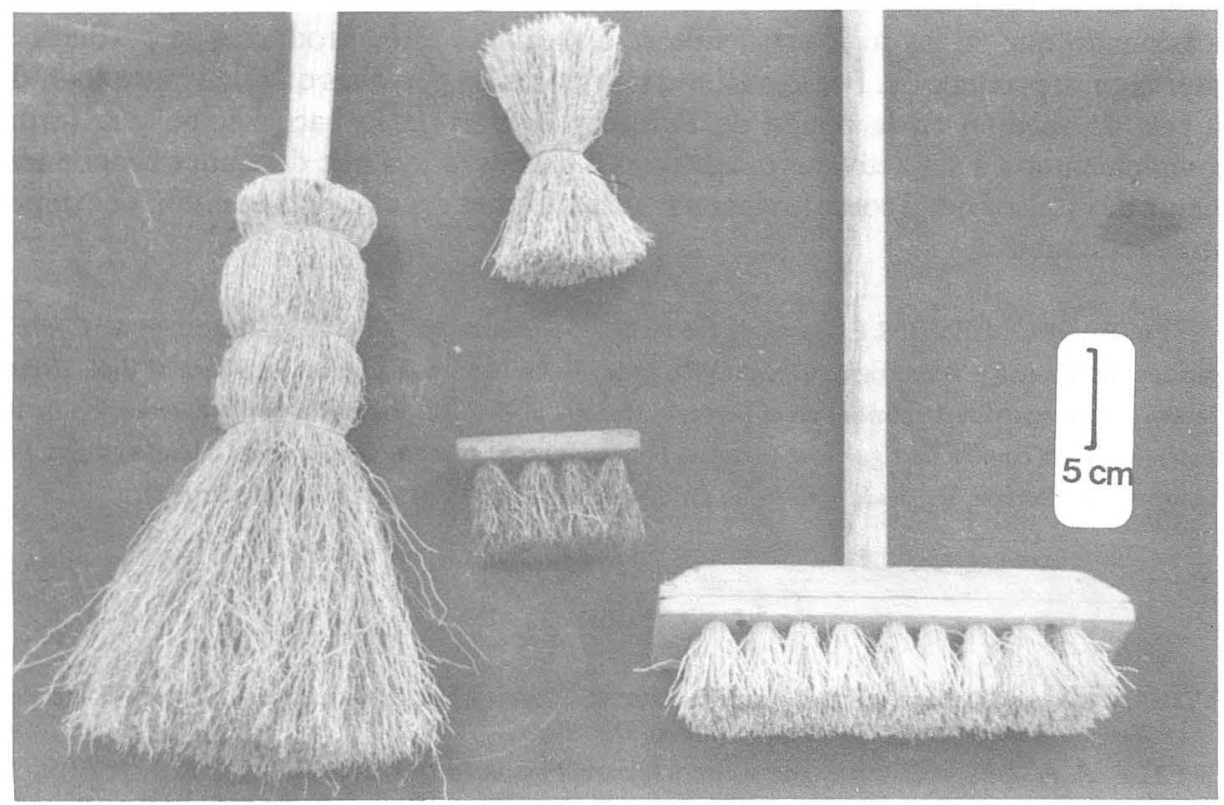

Fig. 3 Productos elaborados con "raíz de zacatón" : escobas, escobetas y cepillos. 
precio de una escobeta varía de acuerdo al tamaño entre \$1000.00 a \$1500.00 (noviembre, 1992) y el de una escoba de $\$ 9500.00$ a $\$ 10000.00$. Para trasladar dicha mercancía a los diferentes mercados se utilizan los autobuses de la región.

En las localidades estudiadas así como en la Región de Perote, cuando el follaje de Muhlenbergia macroura esta verde y tierno se utiliza como forraje para el ganado caprino y equino. En ocasiones también se usa la planta ( $\sin$ raíz) para techar pequeños locales.

Aunque Gerez (1982) cita que la raíz de zacatón fué cultivada para su exportación en 1946, durante el presente estudio no se encontraron indicios de que se continue cultivando esta especie en la región de Perote. Actualmente las personas extraen la raíz de zacatón del medio natural, aunque cada día tienen que dirigirse a lugares más alejados para obtener materia prima de buena calidad, debido a que el zacatón ha sido desplazado por la tala inmoderada y los cultivos (principalmente de papa).

Existen algunos zacatonales en la región de Perote, como los localizados en la carretera PerotePuebla, que se han establecido por efecto del fuego o disturbios ejercidos por tala o pastoreo. Sin embargo, la raíz de estos zacatonales es considerada de mala calidad, debido a que el terreno donde se desarrolla es pedregoso y según las personas entrevistadas es de la denominada "raíz china".

Con la introducción de utensilios domésticos plásticos decayó notablemente la demanda de escobetas, escobas y cepillos elaborados con raíz de zacatón. Esto ha ocasionado que en las regiones donde se elaboraban estos productos se perdiera el intéres en su producción. Por lo cual son muy pocas las personas que siguen explotando la raíz de zacatón en la región de Perote y la mayoría lo hacen como una forma complementaria a sus actividades agrícolas o ganaderas, ya que depender economicamente de esta actividad como lo vienen realizando unas cuantas personas de Tenextepec no es redituable.

AGRADECIMIENTOS. Este trabajo fué realizado pàrcialmente por el apoyo financiero del Consejo Nacional de Ciencia y Tecnología (CONACyT), proyecto 0063N9106. La autora agradece al Biól. Héctor Narave por su contribución inicial en el presente trabajo, al Biól. M. Escamilla por la elaboración de las ilustraciones, así como a las siguientes personas por sus valiosas sugerencias y revisión del manuscrito. Dr. Arturo GómezPompa, Dr. Jesús Valdés R., Dr. Stephen D. Koch y M. en C. Juan J, Ortíz D.

\section{LITERATURA CITADA}

AVILA, H.M. 1980. Explotación de raíz de zacatón. Instituto Nacional de Investigaciones Forestales. Boletín Divulgativo No. 51. SARH. México, D.F. 39p.

BEETLE, A.A. 1997. Noteworthy grasses from Mexico. Phytology 37(4):317407.

GEREZ, F.P. 1982. Historia del uso del suelo en la zona semiárida PoblanaVeracruzana. Tesis, Facultad de Ciencias, UNAM, México, D.F. 98p. 
DÍAZ, G.J.S. 1976. Usos de las plantas medicinales de México. Monografías Científicas II. Instituto

Mexicano para el estudio de las plantas medicinales A.C. México, D.F. 329p.

DÍAZ, P.C.I. 1976. Manual de Gramíneas. UAM, Xochimilco, México, D.F. 345p.

JACKSON, J.R. 1887. Broom root, or Mexican whisk. Gard. Chron. 3(2): 105.

MARTíneZ, M. 1959. Plantas útiles de la Flora Mexicana. Ediciones Botas, México, D.F. 621p.

MARTÍNEZ, M. 1979. Catálogo de nombres vulgares y científicos de plantas mexicanas. Fondo de Cultura Económica. México, D.F. 1220p.

Mejía-Saules, M.T. y P. DÁvila A. 1992. Gramíneas útiles de México. Serie Cuadernos No. 16. Instituto de Biología, UNAM, México, D.F. 298p.

Miranda, F. y HernándeZ X., E. 1963. Los tipos de vegetación de México y su clasificación. Bol. Soc. Bot. de México. 28:29178.

NiETO, P.C. 1985. Catálogo de la flora útil de la Sierra del Ajusco. Secretaría de Agricultura y Recursos Hidráulicos. México, D.F. 17p.

SANDOVAL, J.M.C. 1984. Estudio de la vegetación de la sierra de Mastaloyan, VeracruzPuebla. Tesis, Facultad de Ciencias Biológicas, U.V., Xalapa, Ver. 78p.

SWALLEN, J. 1955. Grasses of Guatemala. En: Flora of Guatemala. Fieldiana, Bot. 24(2):219.

MA. TERESA MEJÍA-SAULES. Instituto de Ecología. Km 2.5 antigua carretera a Coatepec, Apartado Postal 63, 91000 Xalapa, Veracruz. 